\title{
AMS Highlights
}

\section{J. Bedugo ${ }^{a, *}$, on behalf of the AMS Collaboration}

${ }^{a}$ Centro de Investigaciones Energéticas, Medioambientales y Tecnológicas, Av. Complutense 40, Madrid, Spain

E-mail: javier.berdugo@ciemat.es

In ten years on the International Space Station, the Alpha Magnetic Spectrometer (AMS) has collected more than 180 billion cosmic rays measuring with unprecedented precision different components of the charged cosmic rays up to few TeV. This includes fluxes of positrons, electrons, antiprotons, protons, and nuclei from helium to silicon and beyond. A summary of the latest results will be shown. Results on time variation of cosmic ray fluxes associated with solar activity on different time scales will be presented.

37th International Cosmic Ray Conference (ICRC 2021)

July 12 th - 23rd, 2021

Online - Berlin, Germany

${ }^{*}$ Presenter 


\section{Introduction}

The composition and energy spectra of charged cosmic rays are typically interpreted in the context of propagation models [1]. The cosmic ray particles, at least up to about $10^{6} \mathrm{GeV}$, are considered of galactic origin and shock waves of expanding supernovae remnants are good candidates to supply the power for their acceleration. The accelerated particles are injected into the interstellar space and diffused, both in momentum and space, due to interactions with random galactic magnetic fields. Particles are affected by reacceleration, energy losses, nuclear interactions and convective transport during the propagation in the galactic medium. New particles and spallation products are obtained by interaction of cosmic ray particles with the interstellar matter. The understanding of the origin, acceleration and propagation of cosmic rays in the Galaxy require the combination of different measurements over a wide energy range, including chemical composition, anisotropy and solar modulation.

The Alpha Magnetic Spectrometer (AMS) is a general-purpose high-energy particle detector that was deployed to the International Space Station (ISS) on May 19, 2011 to conduct a unique long-duration mission of fundamental physics research from space. Among the scientific goals of AMS are the search for Dark Matter and Antimatter, the study of the propagation of cosmic rays and the exploration of new scientific phenomena that cannot be achieved on ground-based experiments. After ten years of operation in space, AMS has collected more than 180 billion cosmic ray events and provided accurate measurements of electrons, positrons, protons, antiprotons, and nuclei with energies of several $\mathrm{TeV}$ during nearly a complete solar cycle [2]. A compilation of the results of the first seven years of operation has been recently published in Physics Reports [3].

\section{AMS Experiment}

AMS is a magnetic spectrometer designed to identify charged particles up to $\mathrm{Z}=28$ and to measure their energy up to a few $\mathrm{TeV}$ with high accuracy. AMS has been built by an international collaboration that includes institutes from Europe, Asia and America. The core of the detector is a permanent magnet hosting 7 layers of silicon detectors (Tracker) that measure the curvature of the trajectory of the particles and the sign of their charge. The energy loss measured in the silicon sensors is used to determine the absolute charge, with relative resolutions better than $1.5 \%$ for $\mathrm{Z}>8$. Two additional silicon layers are located at the top and bottom of the detector to increase the lever arm in measuring the curvature of high-energy particles and to provide additional measurements of the charge. A time-of-flight (TOF) system is located above and below the magnet. The TOF delivers the main trigger to the detector and measures the velocity and charge of the particles. Below the Lower-TOF, a Ring Imaging Cherenkov Detector (RICH) provides a very accurate measurement of the velocity of the particle (at per mil level) that allows the identification of isotopes up to energies of $\sim 10$ $\mathrm{GeV} /$ nucleon. The Cherenkov detector also performs an independent measurement of the absolute charge of the particles. A transition radiation detector (TRD) on top of the Upper-TOF allows the separation of heavy and light particles. It is a crucial detector for the identification of electrons and positrons from the background of protons. At the bottom of AMS, an electromagnetic calorimeter (ECAL) measures the energy of electromagnetic showers up to $\mathrm{TeV}$ 
energies with accuracies of one percent. The ECAL is a fine granularity 3D calorimeter capable to reconstruct the shape of the showers providing additional information for the identification of electrons and positrons. An array of 16 anti-coincidence counters (ACC) surrounds the Tracker detector inside the magnet bore. Their purpose is to reject particles that enter or exit the tracker volume transversely. A detailed description of the detector and its performances can be found in [3].

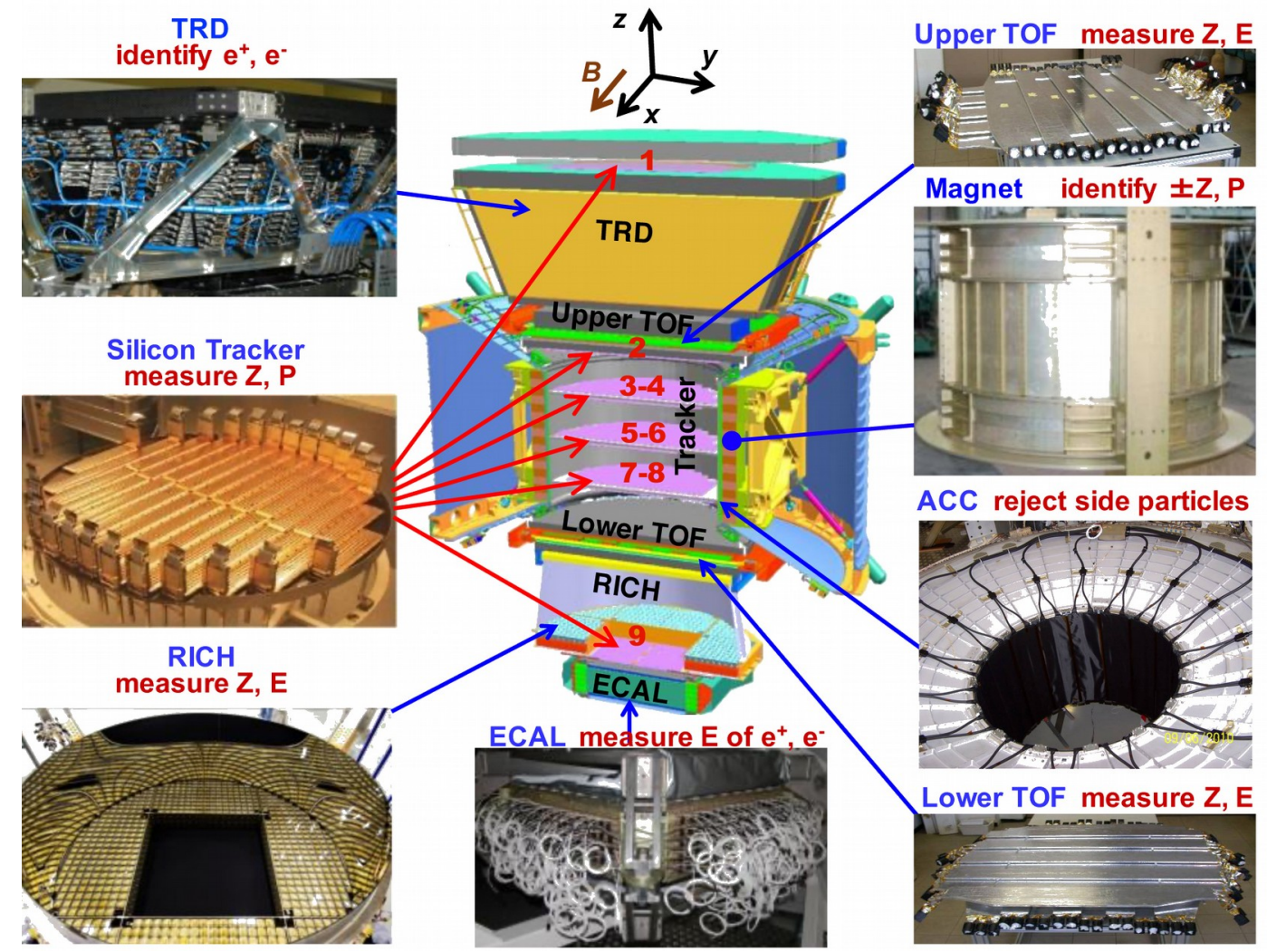

Figure 1: AMS Detector showing the main elements and their functions. The AMS coordinate system, concentric with the magnet, is also shown. The $X$ axis is parallel to the main component of the magnetic field and the $Z$ axis is pointing vertically.

In overall, AMS provides a proton rejection power better than $10^{5}$ for the identification of electrons and positrons up to several hundred $\mathrm{GeV}$ and with very high efficiency. The key feature of AMS is the capability to perform accurate particle identification and charge and momentum measurements from complementary and redundant systems, so that the detector characterization and performances are retrieved from the data itself.

AMS is the first long duration (about two 11-year solar cycles), large acceptance precision magnetic spectrometer to measure the sign and value of the charge, the rigidity (momentum/charge), and the flux of elementary particles, nuclei, and anti-nuclei directly in space.

\section{Properties of cosmic elementary particles}

AMS has reported simultaneous measurements of the fluxes of the charged elementary particles (protons and electrons) and antiparticles (antiprotons and positrons) in cosmic rays [1]. 
These results and the relative behavior of the fluxes over an extended energy range provide accurate experimental information to study the origin, acceleration, and subsequent propagation processes of cosmic rays in the Galaxy. The latest measurements of proton, electron, antiproton and positron fluxes, included in [3], are shown in Fig. 2 and compared with results from other experiments [4], [5], [6], [7].
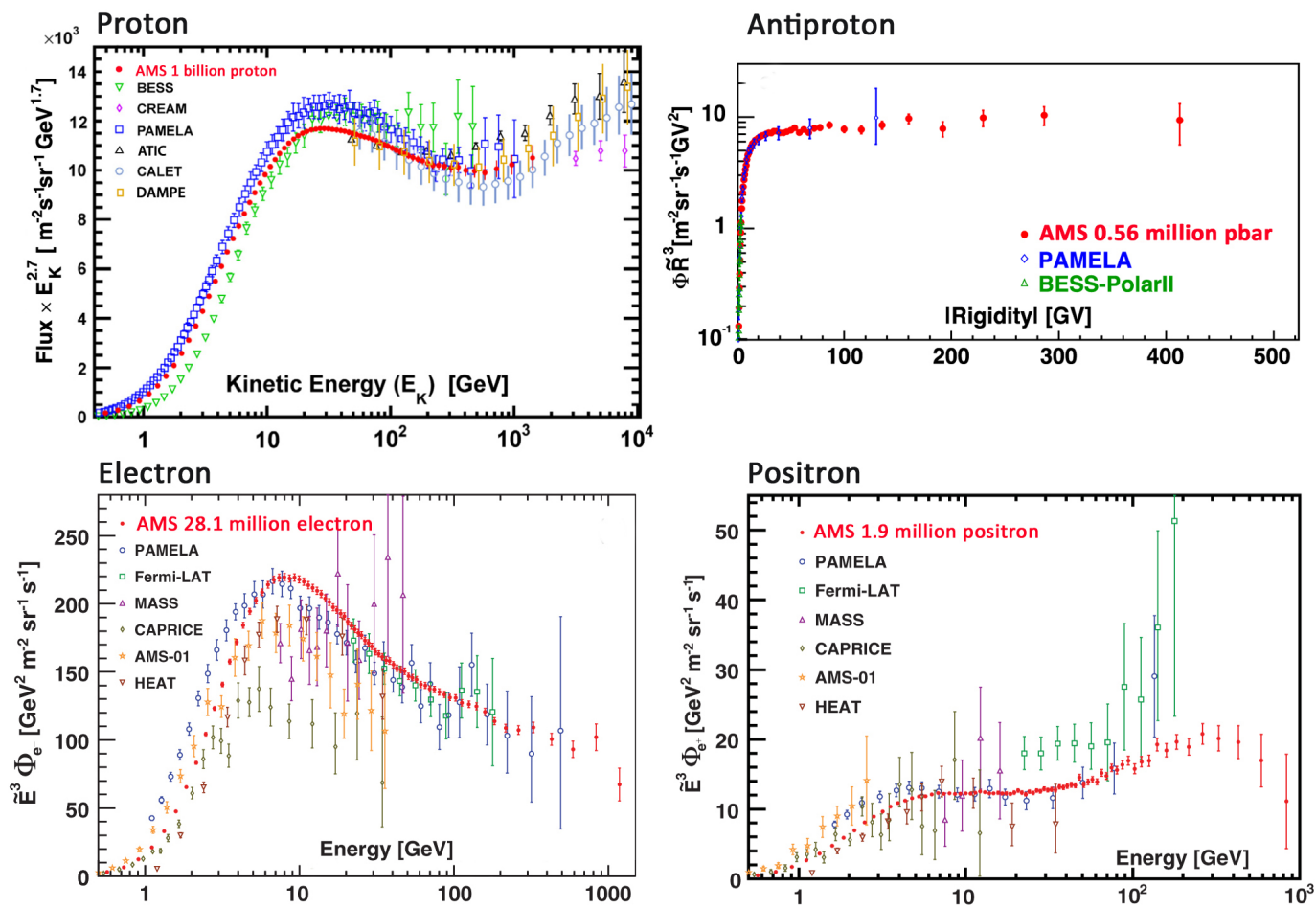

Figure 2: AMS proton, electron, antiproton and positron fluxes together with measurements of other experiments [4], [5], [6, [7]

Electrons and protons are the most abundant elementary particles in the cosmos. The AMS measurement of the proton flux is based on a sample of 1 billion protons, covering an energy range from $0.4 \mathrm{GeV}$ to $1.8 \mathrm{TeV}$, and the electron sample contains 28.1 million electrons, within an energy range from $0.5 \mathrm{GeV}$ to $1.4 \mathrm{TeV}$. A detailed description of the analysis of the proton and electron spectra can be found in [3]. In the entire energy range, the electron energy spectrum can be characterized by a sum of two power law functions, and the proton spectrum is described by a power law function with variable spectral index and progressive hardening above $200 \mathrm{GV}$. As seen in Fig.2, above $\sim 10 \mathrm{GeV}$, electrons exhibit much softer energy dependence compared to protons. This is commonly attributed to energy losses by electrons during the propagation in the interstellar medium.

Measurements of antiprotons and positrons are crucial for the understanding of new phenomena in the cosmos since the yield of these particles from cosmic ray collisions with the interstellar medium is small. The latest AMS measurement of the antiproton flux shown in Fig. 2 extends the rigidity range of our previous measurement up to $525 \mathrm{GV}$ and is based on 0.56 million antiprotons. The positron sample contains 1.9 million positrons and covers the energy range from $0.5 \mathrm{GeV}$ to $1 \mathrm{TeV}$. As seen in Fig. 2, the AMS data significantly extend the antiproton and positron measurements into the uncharted high-energy region. 


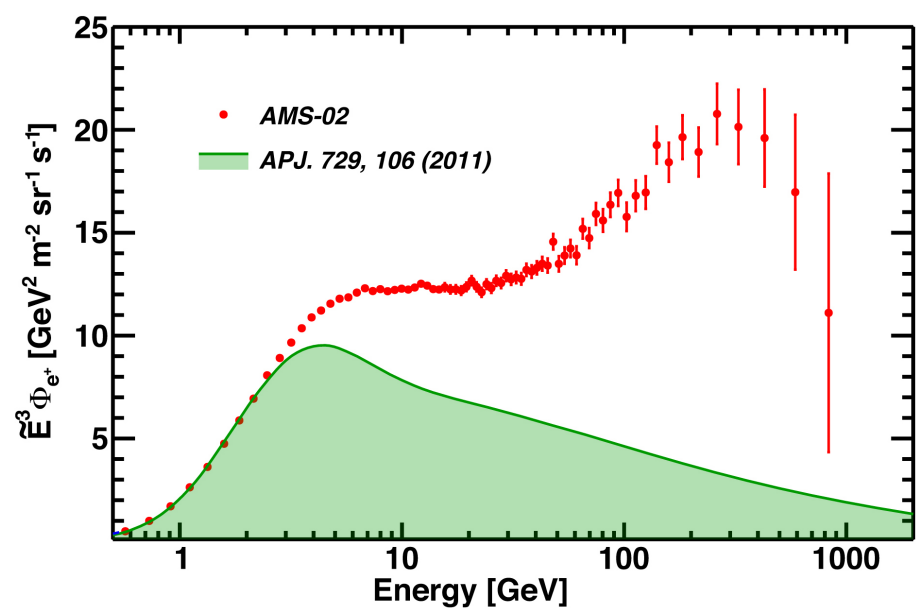

Figure 3: Comparison of the AMS data with a GALPROP model prediction [8] for the secondary positron spectrum (green shaded area). The error bars correspond to the quadratic sum of statistical and systematic errors.

The measured AMS positron spectrum, compared with the GALPROP prediction for secondary production in cosmic ray collisions [8] is shown in Fig. 3. The data presents an increase of the spectrum up to $\sim 25 \mathrm{GeV}$, a maximum at $\sim 284 \mathrm{GeV}$, and the subsequent fall of the observed positron spectrum at higher energies. The complex behavior of the positron flux is not consistent with the exclusive secondary production of positrons and requires the existence of a new source of high energy positrons, either from other astrophysical sources [9] or from annihilation of dark matter particles [10].

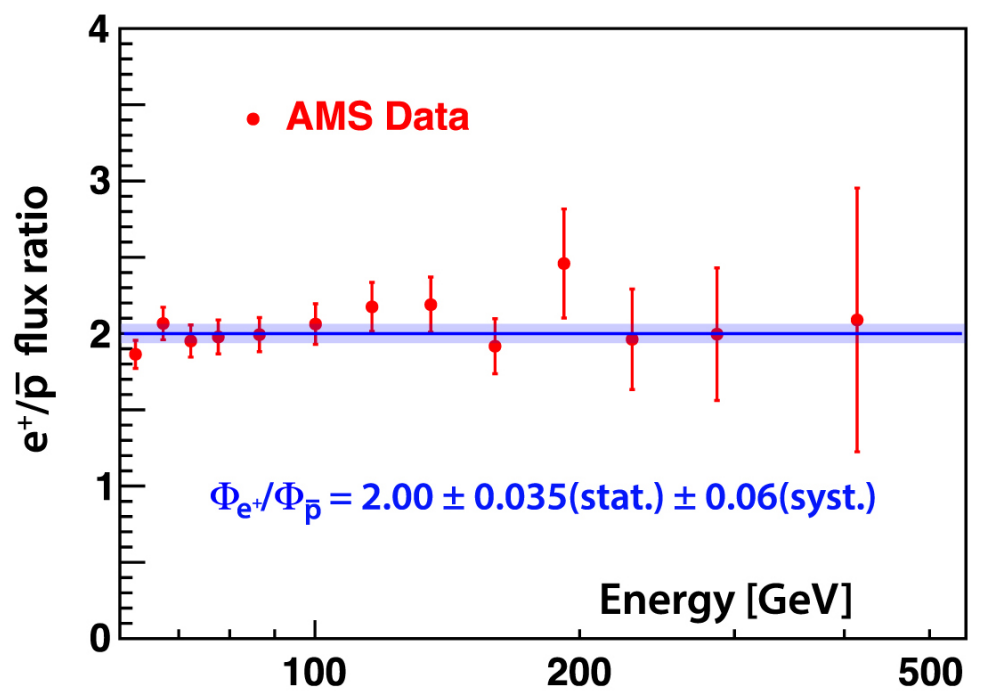

Figure 4: The ratio of the positron-to-antiproton fluxes (red data points) together with the fit of the constant function to data in the energy range [60 - 525] GeV (blue line with 68\% C.L. band).

It is important to note that positron and antiproton spectra have strikingly similar behavior at high energies. Fig. 4 shows the positron-to-antiproton flux ratio above $60 \mathrm{GeV}$. A fit to a constant flux ratio in the range [60 -525$] \mathrm{GeV}$ yields a value of $2.00 \pm 0.035$ (stat.) \pm 
0.06 (syst.), with $\chi^{2} /$ d.o.f. $=7.2 / 12$, consistent with a constant. This might suggest a possible common source of high energy positrons and antiprotons, and then disfavor the pulsar hypothesys for the positron excess since antiprotons are not produced in pulsars. To further study the origin of the positron excess, we have look for anisotropies in the positron sample. New astrophysical sources, like pulsars, may induce some degree of anisotropy in the arrival direction of the particles that, according to some models, could be of the order of $1 \%$. If the excess of positrons has a dark matter origin, it should be more isotropic. From the analysis of the arrival direction of the positrons we conclude that the positron flux is consistent with isotropy. The upper limit on the amplitude of the positron dipole anisotropy, for any axis in galactic coordinates, is $\delta<0.019$ at the $95 \%$ C.L in the energy range from $16 \mathrm{GeV}$ to $500 \mathrm{GeV}$.

By continuing data taking through the lifetime of the ISS (2030) AMS will collect more than 5 million positrons. The increase in the statistics will allow us to extend the measurement of the positron flux up to $2 \mathrm{TeV}$ and to reach sensitivities in the positron anisotropy below the $1 \%$ level expected from pulsars.

\section{Nuclei cosmic rays}

In the context of cosmic ray propagation models, helium, carbon, oxygen and other heavier nuclei produced and accelerated at the astrophysical sources are considered as "primary" cosmic rays. Nuclei produced in interaction of the primaries with the interstellar medium or from the decay of unstable species, are denoted as "secondary" cosmic rays. Considering the energy range between few $\mathrm{GeV} / \mathrm{n}$ and hundreds of $\mathrm{TeV} / \mathrm{n}$, and mainly due to statistical limitations, the accuracy on the measurements from previous experiments does not allow to confirm nor disprove a common spectral index of the primary or secondary cosmic ray nuclei. Measurements of secondary over primary cosmic rays are also affected by backgrounds and uncertainties that limit the precision on the determination of key parameters needed in cosmic ray propagation models.

AMS has measured the fluxes of cosmic ray nuclei from helium $(\mathrm{Z}=2)$ to silicon $(\mathrm{Z}=14)$ as well as the flux of iron $(Z=26)$, the heaviest stable nuclei from stellar nucleosynthesis. AMS measurements cover a rigidity range from few GV to 1-3 TV with accuracies of the order of few percent at $100 \mathrm{GV}$. A compilation of the rigidity dependence of the fluxes of cosmic ray nuclei from $Z=2$ to $Z=14$ measured by AMS are presented in Fig. 5 . Their main characteristics are discussed below:

\subsection{Primary cosmic ray nuclei}

Helium ( $\mathrm{Z}=2)$, carbon $(\mathrm{Z}=6)$, and oxygen $(\mathrm{Z}=8)$ are among the most abundant nuclei in cosmic rays. The AMS He, $\mathrm{C}$ and $\mathrm{O}$ flux measurements reported in [3] are based on samples of 125 million helium, 14 million carbon and 12 million oxygen nuclei and show that the fluxes deviate from a single power law and their spectral indices progressively harden above $200 \mathrm{GV}$. As illustrated in Fig. 5, above 60 GV, He, C and O fluxes have identical rigidity dependence. 


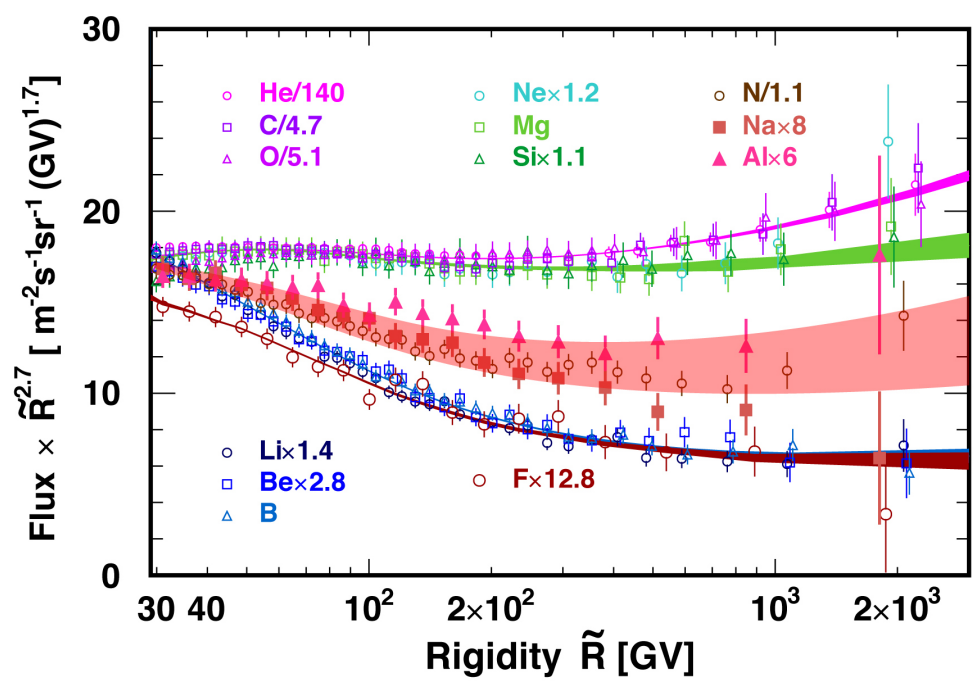

Figure 5: The fluxes of cosmic nuclei, from $Z=2$ to $Z=14$, measured by AMS as a function of rigidity above $30 \mathrm{GV}$. For display purposes only, fluxes were rescaled as indicated. The shaded tan band on $\mathrm{N}, \mathrm{Na}$, and $\mathrm{Al}$ is to guide the eye.

Recently, AMS has reported the measurements of neon $(\mathrm{Z}=10)$, magnesium $(\mathrm{Z}=12)$ and silicon $(Z=14)$ fluxes based on samples of 1.8 million, 2.2 million and 1.6 million nuclei respectively [2]. The results show that $\mathrm{Ne}, \mathrm{Mg}$ and $\mathrm{Si}$ have identical rigidity dependence above 86.5 GV and, similarly as for He, C and O, they deviate from a single power law above $200 \mathrm{GV}$. Unexpectedly, above $86.5 \mathrm{GV}$ the rigidity dependence of Ne-Mg-Si spectra is different from the rigidity dependence of He-C-O. The AMS results show that primary cosmic rays have at least two distinct classes of rigidity dependence.

\subsection{Secondary cosmic ray nuclei}

Lithium $(\mathrm{Z}=3)$, beryllium $(\mathrm{Z}=4)$, and boron $(\mathrm{Z}=5)$ nuclei in cosmic rays are considered to be produced by the collisions of heavier nuclei (as carbon and oxygen) with the interstellar medium. AMS measurement of the Li, Be and B fluxes reported in [3] are based on 3.0 million, 1.7 million, and 4.2 million nuclei respectively. The AMS measurements show that all three fluxes have identical rigidity dependence above $30 \mathrm{GV}$. The three fluxes deviate from a single power law above $200 \mathrm{GV}$ in an identical way but their rigidity dependence is distinctly different from the primary $\mathrm{He}, \mathrm{C}$ and $\mathrm{O}$ cosmic rays. Fluorine $(\mathrm{Z}=9)$ is the only purely secondary cosmic ray nuclei between oxygen and silicon and is thought to be produced mostly by the collisions of $\mathrm{Ne}, \mathrm{Mg}$, and $\mathrm{Si}$, with the interstellar medium.

AMS has recently reported the accurate measurement of the fluorine flux in cosmic rays based on 0.29 million nuclei collected by AMS during the first 8.5 years of operation [2]. As seen from Fig. 5, the fluorine spectrum deviates from a single power law above $200 \mathrm{GV}$. At high rigidities, above $175 \mathrm{GV}$, the rigidity dependence of the fluorine flux is identical to the rigidity dependence of the light secondary cosmic ray nuclei ( $\mathrm{Li}, \mathrm{Be}$ and $\mathrm{B}$ ) and at low rigidities, they are different. 


\subsection{Cosmic ray nuclei with primary and secondary components}

Nitrogen $(Z=7)$, sodium $(Z=11)$ and aluminum $(Z=13)$ cosmic rays are produced in astrophysical sources and by collisions of heavier nuclei with the interstellar medium. Therefore, their fluxes are expected to contain both, primary and secondary components. AMS has reported the measurement of the flux of nitrogen nuclei in cosmic rays and, more recently, the measurements of the fluxes of sodium and aluminum nuclei [2]. AMS measurements are based on samples of 3.9 million nitrogen, 0.46 million sodium and 0.51 million aliuminum nuclei. As seen in Fig 5, the nitrogen, sodium and aluminum flux spectra present a different rigidity dependence than primary and secondary cosmic ray nuclei and belong to a distinct group.
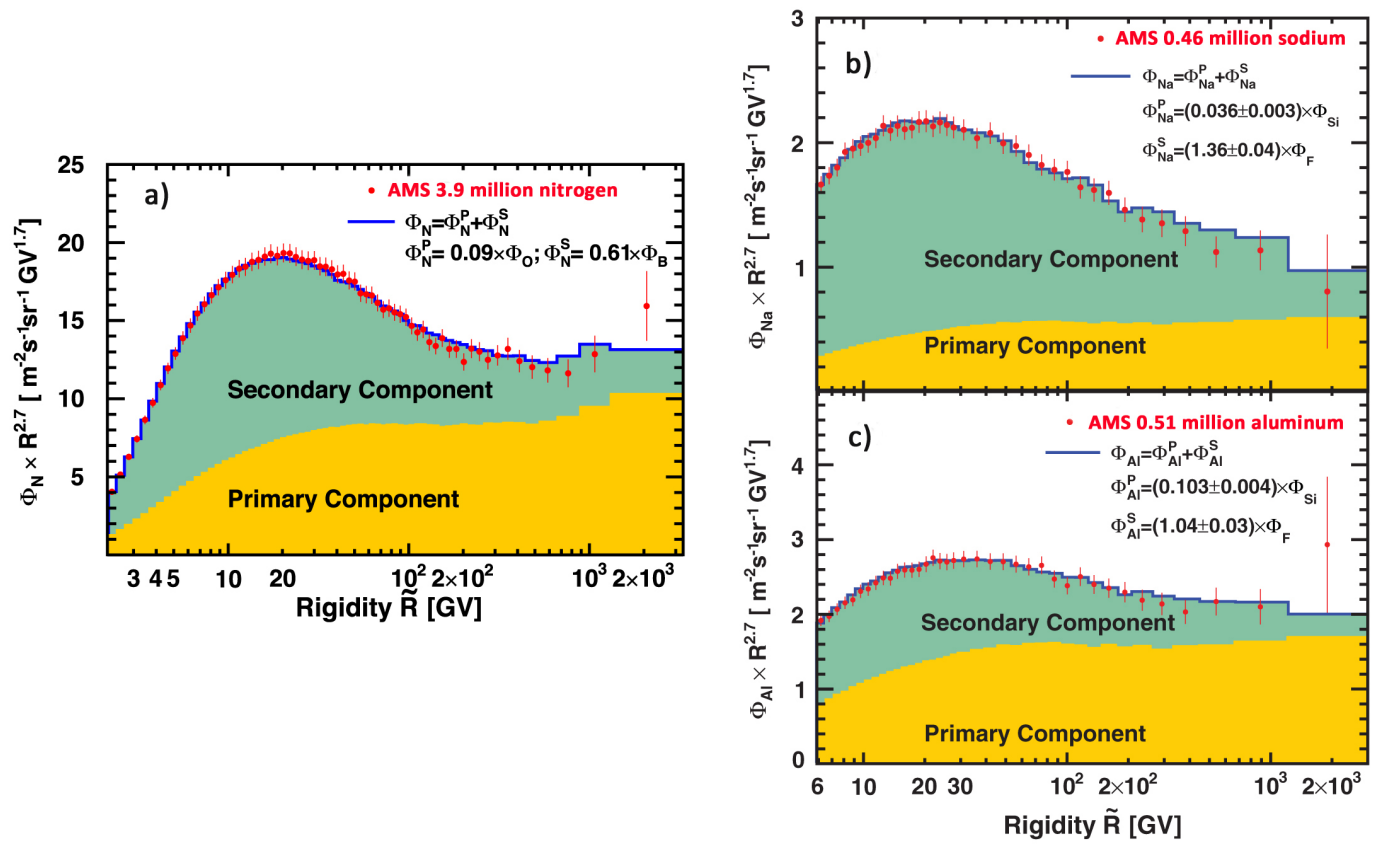

Figure 6: (a) The AMS nitrogen, (b) sodium and (c) aluminum fluxes fitted as weighted sums of primary and secondary fluxes. The fits yield $\chi^{2} /$ dof of 59/64 for nitrogen, 19/36 for sodium and 24/36 for aluminum. The contributions of the primary and secondary components are indicated by the shading areas (yellow and green, respectively).

To obtain the fraction of the primary and secondary components in $\mathrm{N}, \mathrm{Na}$ and $\mathrm{Al}$ fluxes, the measured values have been fitted to linear combinations of primary and secondary cosmic ray fluxes as function of the rigidity (see Fig. 6). In the case of nitrogen nuclei, the AMS measured fluxes of oxygen and boron are taken as characteristic primary and secondary fluxes. For sodium and aluminum, the measured silicon and fluorine fluxes are used. As shown in Fig. 6 , the fits reproduce very well the nitrogen, sodium and aluminum fluxes in a wide rigidity range. The fractions of the primary component increases with rigidity for the three nuclei and becomes dominant at the highest rigidities. The observation that $\mathrm{N}, \mathrm{Na}$ and $\mathrm{Al}$ fluxes is well described as linear combinations of primary and secondary fluxes over a wide rigidity range permits the direct determination of the $\mathrm{N} / \mathrm{O}, \mathrm{Na} / \mathrm{Si}$ and $\mathrm{Al} / \mathrm{Si}$ abundance ratios at the source $(0.092 \pm 0.002$ for $\mathrm{N} / \mathrm{O}, 0.036 \pm 0.003$ for $\mathrm{Na} / \mathrm{Si}$ and $0.103 \pm 0.004$ for $\mathrm{Al} / \mathrm{Si})$ without the need to consider the Galactic propagation of cosmic rays. 


\subsection{Secondary-to-primary flux ratios}

The precise measurements of the rigidity dependence of primary and secondary cosmic rays provide key information on propagation and source processes. Fig. 7 shows, as a function of the rigidity, the ratio of the light secondary nuclei fluxes ( $\mathrm{Li}-\mathrm{Be}-\mathrm{B})$ with respect to the primary oxygen and carbon nuclei flux. The distributions can be characterized by a broken power law at $\sim 200 \mathrm{GV}$ with similar spectral indices $\Delta_{1}$ and $\Delta_{2}$ for the three ratio of fluxes., as shown in Fig. 8. The average hardening is $\Delta_{2}$ [192-3300] GV $-\Delta_{1}[60.3-192] \mathrm{GV}=0.140 \pm$ 0.025 , showing that at high rigidities the secondary cosmic rays harden more than the primary ones with a significance bigger than $5 \sigma$. This additional hardening of secondary cosmic rays is consistent with expectations when the hardening of the individual fluxes is due to the propagation in the Galaxy.
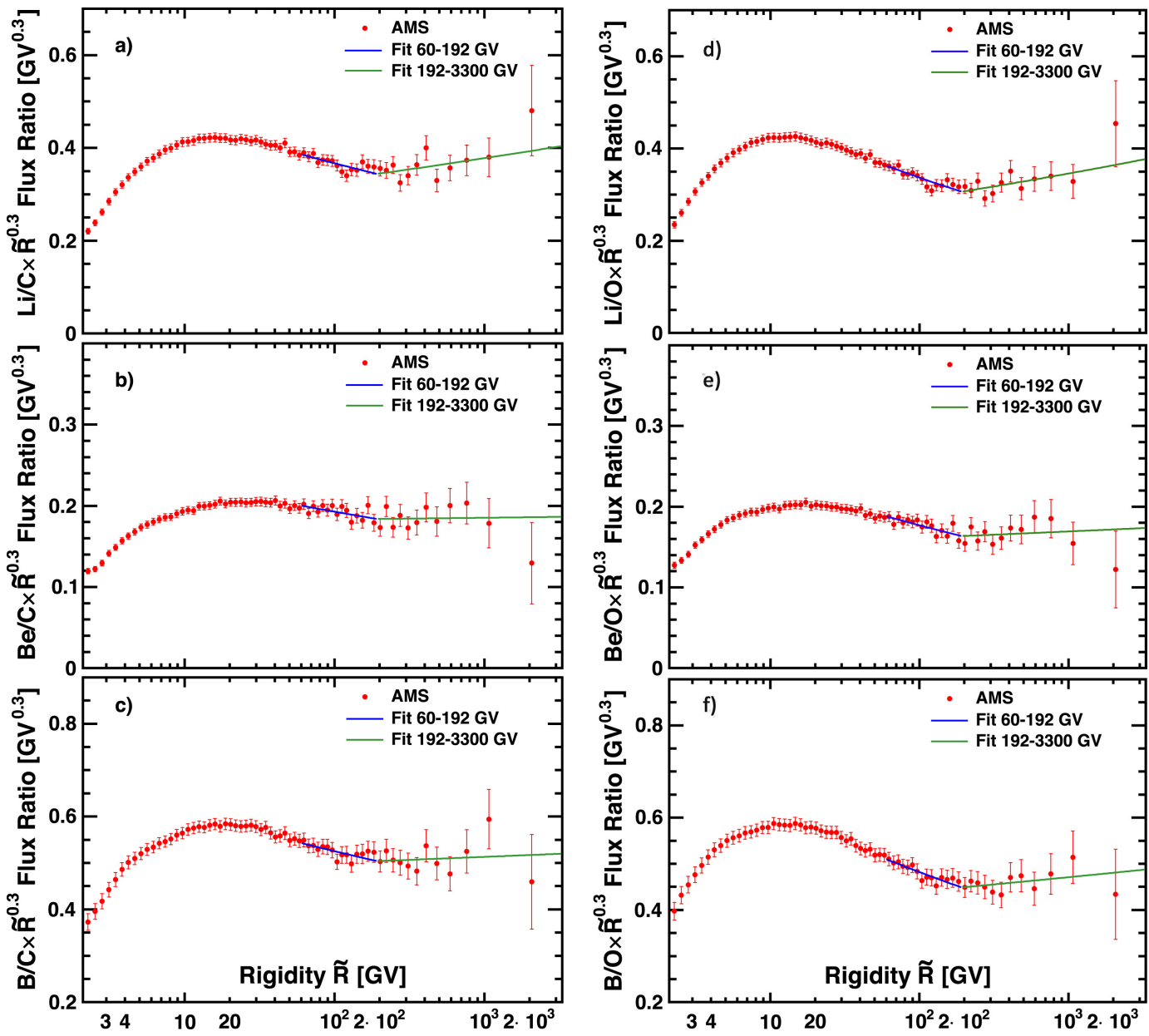

Figure 7: The AMS secondary to primary flux ratios: (a) Li/C, (b) Be/C, (c) B/C, (d) Li/O, (e) $B e / O$, (f) B/O. For display purposes, the ratios are scaled with $R^{0.3 .}$ Solid lines show the fits of single power law function $\left(\mathrm{CR}^{\Delta}\right)$ to data in two non-overlapping intervals [60.3 - 192] (blue) and [192 - 3300]GV (green) 

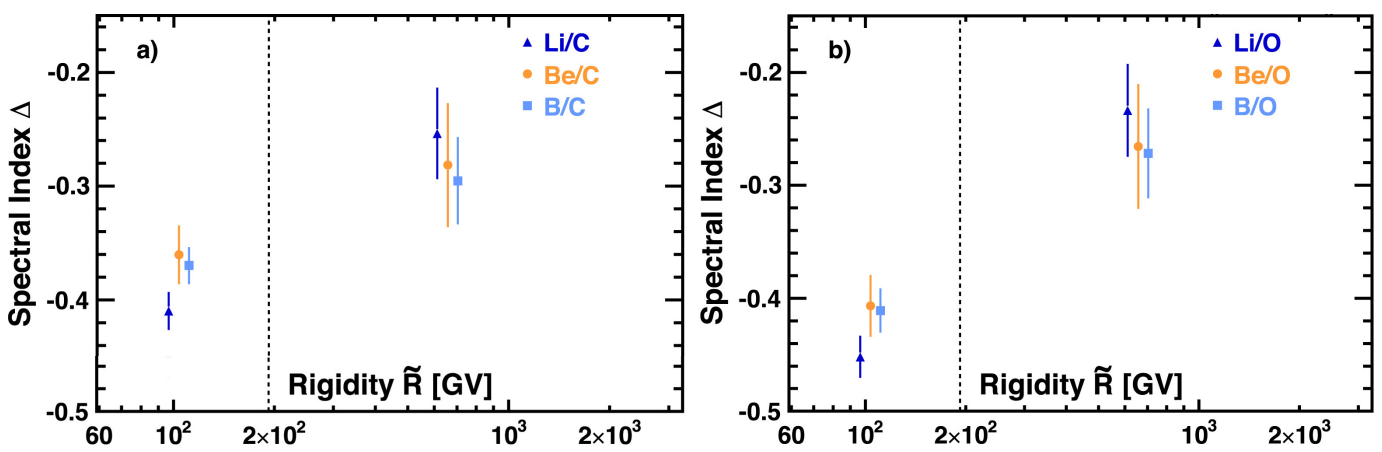

Figure 8: Spectral index $\Delta$, determined from the fits of single power law function $\left(C R^{\Delta}\right)$ to data in two non-overlapping intervals [60.3 - 192] and [192 - 3300]GV.

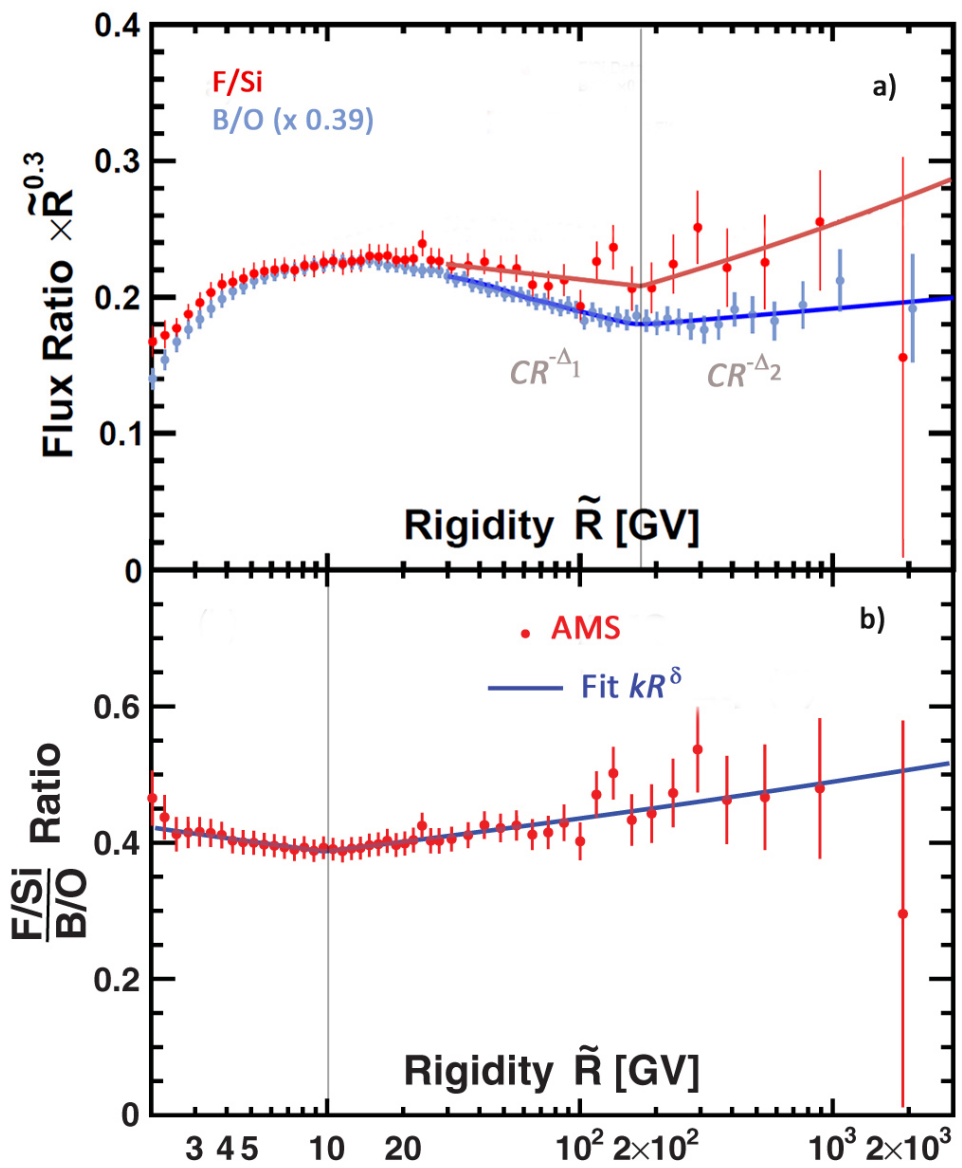

Figure 9: (a) The AMS F/Si flux ratio (red dots) and AMS B/O flux ratio (blue dots) as a function of rigidity with total errors. For display purposes only, the F/Si and B/O flux ratios are multiplied by $R^{0.3}$ and the $B / O$ flux ratio rescaled as indicated. The solid brown and blue curves show the F/Si and B/O fit results with single power law functions $\left(\mathrm{CR}^{-\Delta}\right)$, respectively. (b) The AMS $[(\mathrm{F} / \mathrm{Si}) /(\mathrm{B} / \mathrm{O})]$ ratio as a function of rigidity with total errors. The solid blue curve shows the fit results to the function $k R^{\delta}$.

Traditionally the light secondary-to-primary ratio $\mathrm{B} / \mathrm{O}$ (or $\mathrm{B} / \mathrm{C}$ ) is used to describe the propagation properties of all cosmic rays. Fig. 9(a) shows the rigidity dependence of the heavier 
secondary-to-primary F/Si flux ratio together with the AMS flux ratio B/O. As seen, although the $\mathrm{F} / \mathrm{Si}$ flux ratio hardens above $175 \mathrm{GV}$ in a similar way as $\mathrm{B} / \mathrm{O}$, the heavier secondary-toprimary $\mathrm{F} / \mathrm{Si}$ flux ratio rigidity dependence is distinctly different from the lighter $\mathrm{B} / \mathrm{O}$ rigidity dependence. In particular, as shown in Fig. 9(b), above $10 \mathrm{GV}$, the $[(\mathrm{F} / \mathrm{Si}) /(\mathrm{B} / \mathrm{O})]$ ratio can be described by a power law $\mathrm{R}^{\delta}$ with $\delta=0.052 \pm 0.007$ (a $7 \sigma$ difference from zero). This indicates that the propagation properties of heavy cosmic rays, from $\mathrm{F}$ to $\mathrm{Si}$, are different from those of light cosmic rays, from He to $\mathrm{O}$.

\subsection{Iron Flux}

Primary iron $(\mathrm{Z}=26)$ cosmic rays are the heaviest stable nuclei from stellar nucleosynthesis. Iron interaction cross sections with the interstellar medium (p, He) are significantly larger than those of lighter nuclei ( $\mathrm{He}, \mathrm{C}, \mathrm{O}, \mathrm{Ne}, \mathrm{Mg}$, and $\mathrm{Si}$ ). Therefore, iron nuclei interact much more with the interstellar medium during propagation. The rigidity dependence of the iron flux compared with that of lower-charge primary cosmic rays provides new insights into the origin and propagation of cosmic rays.
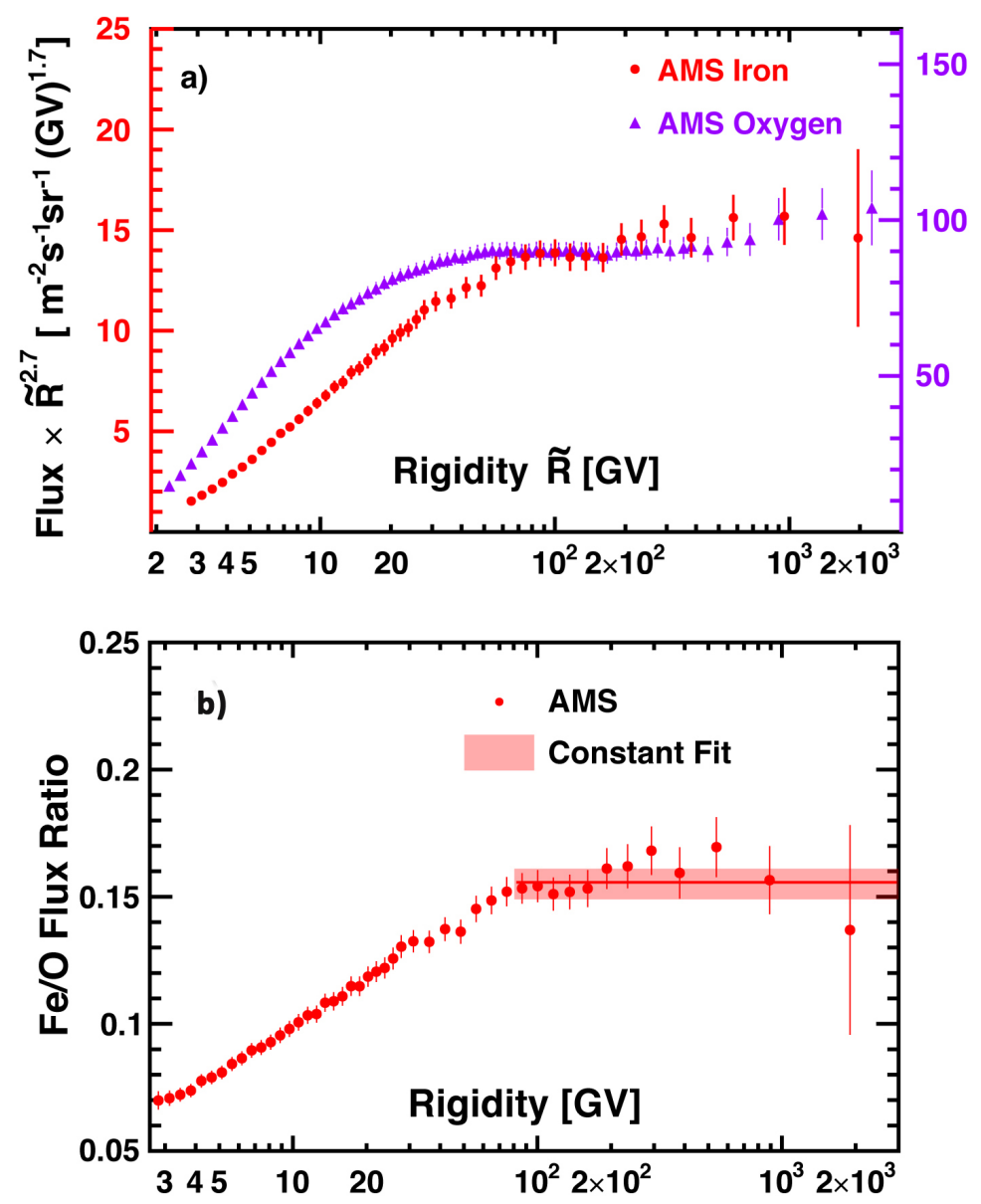

Figure 10: (a) The AMS iron flux (red dots) and oxygen flux (violet triangles) multiplied by $R^{2.7}$ with total errors as a function of rigidity. (b) The AMS Fe/O ratio as a function of rigidity

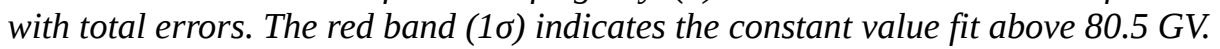


Figure 10 (a) shows the AMS measurement of the iron nuclei cosmic ray flux in the rigidity range $2.65 \mathrm{GV}$ to $3.0 \mathrm{TV}$ based on 0.62 million iron nuclei [2]. The results are compared with the AMS measurement of oxygen nuclei flux. Above $80.5 \mathrm{GV}$ the rigidity dependence of the Fe flux is identical to the rigidity dependence of the light primary cosmic ray $\mathrm{He}, \mathrm{C}$, and $\mathrm{O}$ fluxes, with the $\mathrm{Fe} / \mathrm{O}$ flux ratio being constant at $0.155 \pm 0.006$ as shown in Fig 10 (b). Unexpectedly $\mathrm{Fe}$ and $\mathrm{He}, \mathrm{C}$, and $\mathrm{O}$ belong to the same class of primary cosmic rays which is different from the primary cosmic rays $\mathrm{Ne}, \mathrm{Mg}$, and Si class.

\subsection{Isotopes}

Isotopes of light nuclei provide complementary constraints on the propagation parameters compared to those obtained from secondary nuclei ( $\mathrm{Li}, \mathrm{Be}$ and $\mathrm{B}$ ). This is particularly interesting in the case of ${ }^{2} \mathrm{H}$ and ${ }^{3} \mathrm{He}$ isotopes which are mostly produced by the fragmentation of the primary ${ }^{4} \mathrm{He}$ with the interstellar medium, whereas $\mathrm{Li}$, Be, and $\mathrm{B}$ are produced by multiple fragmentation channels of many heavy nuclei with the interstellar medium. In addition, the comparison of ${ }^{2} \mathrm{H} /{ }^{4} \mathrm{He}$ and ${ }^{3} \mathrm{He} /{ }^{4} \mathrm{He}$ ratios with those from heavier nuclei constitutes a test of the diffusion properties at different distances since ${ }^{2} \mathrm{H},{ }^{3} \mathrm{He}$ and ${ }^{4} \mathrm{He}$ interaction cross sections with the interstellar medium are significantly smaller than those of heavier nuclei and travels larger distances.

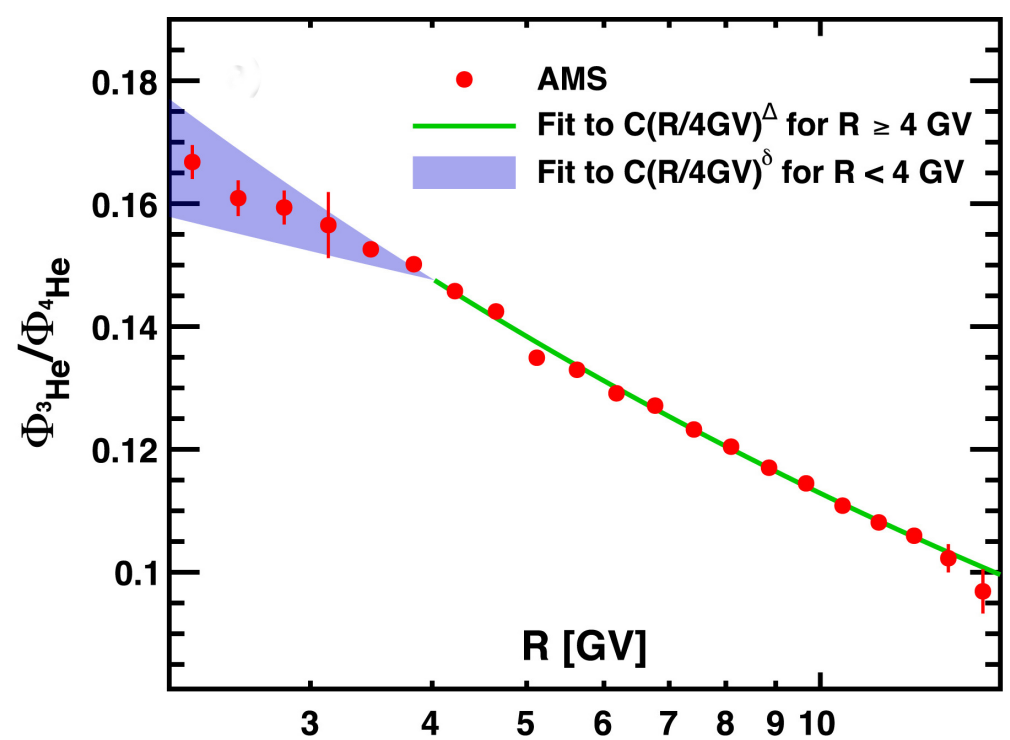

Figure 11: The time-averaged ${ }^{3} \mathrm{He}{ }^{4} \mathrm{He}$ flux ratio as a function of rigidity with statistical and uncorrelated systematic errors added in quadrature. The solid green curve shows a single power law fit $C(R / 4 G V)^{4}$ above $4 G V$. The shaded blue area indicates the results of single power law fits to $C(R / 4 G V)^{\delta}$ below $4 \mathrm{GV}$ for each of the 21 time periods.

AMS has reported in 2019 the precision measurements of the cosmic-ray ${ }^{3} \mathrm{He}$ and ${ }^{4} \mathrm{He}$ isotope fluxes and their ratio as functions of rigidity from 1.9 to $15 \mathrm{GV}$ for ${ }^{3} \mathrm{He}$, from 2.1 to 21 GV for $4 \mathrm{He}$, and from 2.1 to $15 \mathrm{GV}$ for the ${ }^{3} \mathrm{He} /{ }^{4} \mathrm{He}$ flux ratio [2]. The measurement is based on 100 million ${ }^{4} \mathrm{He}$ and 18 million ${ }^{3} \mathrm{He}$ nuclei collected by AMS from May 2011 to November 2017 and has been done in 21 time periods of four Bartels rotations (108 days) each, ensuring enough statistics to measure ${ }^{3} \mathrm{He}$ to percent level accuracy. Fig. 11 shows the time-averaged ${ }^{3} \mathrm{He} /{ }^{4} \mathrm{He}$ flux ratio as function of rigidity. Above $4 \mathrm{GV}$, the ${ }^{3} \mathrm{He} /{ }^{4} \mathrm{He}$ flux ratio is time independent and is 
well described with a single power law $\left(\mathrm{CR}^{\Delta}\right)$ with $\Delta=-0.294 \pm 0.004$, in good agreement with the Kolmogorov theory of turbulence which predicts $\Delta=-1 / 3$ asymptotically. Below $4 \mathrm{GV}$, the ${ }^{3} \mathrm{He} /{ }^{4} \mathrm{He}$ flux ratio is time dependent. To study the time and rigidity dependence of the ${ }^{3} \mathrm{He} /{ }^{4} \mathrm{He}$ flux ratio, fits to $\mathrm{CR}^{\delta}$ for each time period of four Bartels rotations were performed. The results of the fits are shown as a shaded band in Fig. 11 and yield an average $\delta$ of $-0.21 \pm 0.02$ with a time variation of \pm 0.05 .

AMS has also measured the cosmic ray fluxes of deuteron $\left({ }^{2} \mathrm{H}\right)$ and the isotopes of lithium $\left({ }^{6} \mathrm{Li},{ }^{7} \mathrm{Li}\right)$ and beryllium $\left({ }^{7} \mathrm{Be},{ }^{9} \mathrm{Be},{ }^{10} \mathrm{Be}\right)$ which will be published by the Collaboration in the near future.

\section{Solar physics}

Cosmic rays from the interstellar medium are screened by the heliosphere. This is particularly visible at low energies. To properly uncover the properties of the local interstellar spectrum of cosmic rays, a deep understanding of the solar modulation effects is needed.

Cosmic ray transport in the heliosphere is rigidity dependent and related to changes in solar activity. The dynamics of the interplanetary environment induces temporal changes in cosmic rays that correlate with solar activity at different time scales. The most significant longterm scale variation is the 11-year solar cycle during which the number of sunspots changes from minimum to maximum and then back to a minimum. Shorter scale variations can be either non-recurrent or recurrent. The non-recurrent variations are mainly due to the interactions of cosmic rays with strong transient disturbances in the interplanetary magnetic field, such as interplanetary coronal mass ejections, e.g., shock waves, especially during solar maxima, that can last from days to weeks. Recurrent variations with a period of 27 days, corresponding to the synodic solar rotation, and at multiples of that frequency (e.g., periods of 13.5 and 9 days) are related to the passage of corotating interaction regions originating from one or more coronal holes of the Sun. Measuring the time evolution of cosmic ray fluxes with different particles over an extended period of time covering an extended rigidity range is very valuable input to study the solar modulation effects.

AMS started taking data in 2011 and the period of observation has covered almost one complete solar cycle which includes the period of Sun magnetic field reversal. Previously, AMS has reported the time evolution of the monthly proton, helium, electrons and positrons fluxes measured during the first 7 years of data taking [2].

New AMS results on the time evolution of the proton and helium fluxes in a daily basis are presented in Fig. 12. The daily flux measurements, with accuracies of 1-3\%, cover an extended period of time (8.5 years) and the samples contain a total of 5.5 billion proton events measured in the rigidity range from $1 \mathrm{GV}$ to $100 \mathrm{GV}$ and 0.76 billion helium events from $1.71 \mathrm{GV}$ to 100 GV. As seen, both particle fluxes exhibit variations on different time scales, from days to years. Long-term variation related to the 11-year solar cycle is clearly visible as well as recurrent and non-recurrent flux variations at different rigidity bins. The results and detailed analysis of the AMS daily proton and daily helium fluxes from 1 to $100 \mathrm{GV}$ will be published in forthcoming publications. 


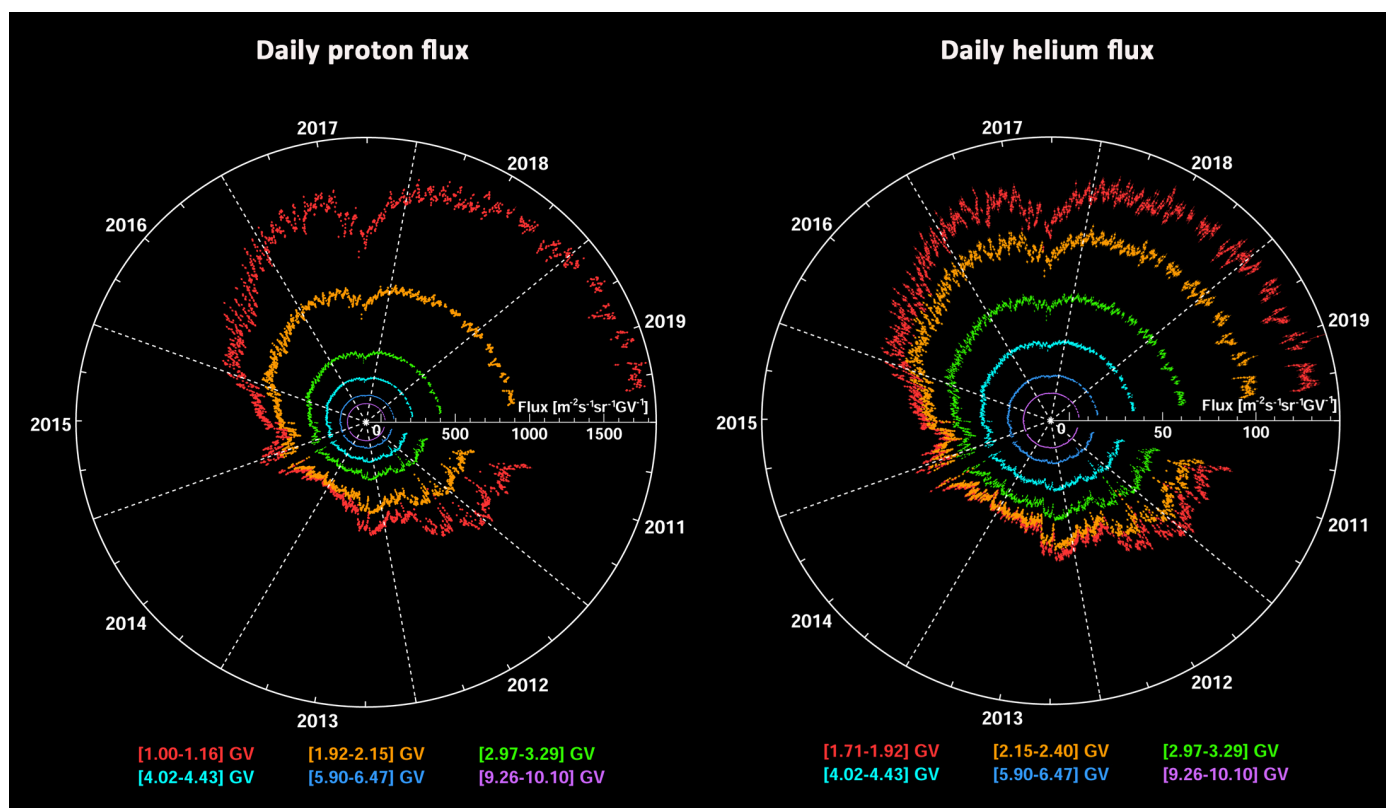

Figure 12: The AMS daily proton and daily helium fluxes for six typical rigidity bins measured from May 20, 2011 to October 29, 2019 which includes a major portion of Solar Cycle 24 (from December 2008 to December 2019).

\section{Summary remarks}

AMS is the first and only instrument providing simultaneous measurements of particles, anti-particles and chemical composition of cosmic ray nuclei in an extended energy range and over a solar cycle. In ten years on the ISS, AMS has recorded more than 180 billion cosmic rays and the precision and characteristics of the AMS data provide unique input to the understanding of cosmic ray production and propagation. As an external payload on the ISS until at least 2030, AMS will continue to collect and analyze an increasing set of data at highest energies which will provide new insights in this field and will allow to continue exploring new physics and astrophysics phenomena.

\section{References}

[1] I.A. Grenier, J.H. Black, A.W. Strong, Annu. Rev. Astron. Astrophys. 53 (2015) 199; P. Blasi, Astron. Astrophys. Rev. 21 (2013) 70; A.W. Strong, I.V. Moskalenko, V.S. Ptuskin, Annu. Rev. Nucl. Part. Sci. 57 (2007) 285.

[2] A compilation of AMS publications can be found at. https://ams02. space/publications.

[3] M. Aguilar et al. (AMS Collaboration). The Alpha Magnetic Spectrometer (AMS) on the International Space Station: Part II - Results from the First Seven Years. Phys. Rep. 894, 1 (2021).

[4] We are grateful to the BESS-Polar Collaboration for providing the BESS-Polar II proton spectrum, measured during a 25-day Antarctic balloon flight in December 2007 and January 2008. Statistical and systematic errors are combined. A publication detailing these measurements is in preparation. 
For a description of the BESS-Polar experiment see K. Abe et al., Phys. Rev. Lett. 108, 051102 (2012).

For the CREAM experiment see Y. S. Yoon et al., Astrophys. J. 728, 122 (2011).

For the PAMELA experiment see O. Adriani et al., Astrophys. J. 765, 91 (2013); Science 332, 69 (2011).

For the ATIC experiment see A. D. Panov et al., Bull. Russ. Acad. Sci. Phys. 73, 564 (2009)

For the CALET experiment see O. Adriani, et al., Phys. Rev. Lett. 122 (2019) 181102.

For the DAMPE experiment see Q. An, et al., Sc. Adv. 5 (9) (2019) eaax3793.

[5] For PAMELA see O. Adriani, et al., Phys. Rev. Lett. 106 (2011) 201101.

For Fermi-LAT see M. Ackermann, et al., Phys. Rev. Lett. 108 (2012) 011103.

For MASS see C. Grimani, et al., Astron. Astrophys. 392 (2002) 287.

For CAPRICE see M. Boezio, et al., Adv. Space Res. 27 (2001) 669.

Form AMS-01 see J. Alcaraz, et al., Phys. Lett. B 484 (2000) 10.

For HEAT see S.W. Barwick, et al., Astrophys. J. 498 (1998) 779; M.A. DuVernois, et al., Astrophys. J. 559 (2001) 296; and J.J. Beatty, et al., Phys. Rev. Lett. 93 (2004) 241102.

[6] For Bess-Polar II see K. Yoshimura, et al., Phys. Rev. Lett. 75 (1995) 3792; S. Orito, et al., Phys. Rev. Lett. 84 (2000) 1078; Y. Asaoka, et al., Phys. Rev. Lett. 88 (2002) 051101; K. Abe, et al., Phys. Lett. B 670 (2008) 103; K. Abe, et al., Phys. Rev. Lett. 108 (2012) 051102; K. Abe, et al., Astrophys. J. 882 (2016) 65.

For PAMELA see O. Adriani, et al., Phys. Rev. Lett. 102 (2009) 051101; O. Adriani, et al., Phys. Rev. Lett. 105 (2010) 121101; O. Adriani, et al., JETP Lett. 96 (2013) 621.

[7] For PAMELA see O. Adriani, et al., Phys. Rev. Lett. 111 (2013) 081102.

For Fermi-LAT see M. Ackermann, et al., Phys. Rev. Lett. 108 (2012) 011103.

For MASS see C. Grimani, et al., Astron. Astrophys. 392 (2002) 287.

For CAPRICE see M. Boezio, et al., Adv. Space Res. 27 (2001) 669.

Form AMS-01 see M. Aguilar, et al., Phys. Lett. B 646 (2007) 145.

For HEAT see S.W. Barwick, et al., Astrophys. J. 498 (1998) 779; M.A. DuVernois, et al., Astrophys. J. 559 (2001) 296; and J.J. Beatty, et al., Phys. Rev. Lett. 93 (2004) 241102.

[8] FR. Trotta, G. Johannesson, I. Moskalenko, T. Porter, R. Ruiz de Austri, A. Strong, Astrophys. J. 729 (2011) 106.

[9] P.D. Serpico, Astropart. Phys. 39-40 (2012) 2;T.

Linden, S. Profumo, Astrophys. J. 772 (2013) 18;

P. Mertsch , S. Sarkar, Phys. Rev. D 90 (2014) 061301;

N. Tomassetti, F. Donato, Astrophys. J. Lett. 803 (2015) L15;

D. Hooper, I. Cholis, T. Linden, K. Fang , Phys. Rev. D 96 (2017) 103013;

W. Liu, X.J. Bi, S.J. Lin, B.B. Wang, P.F. Yin, Phys. Rev. D 96 (2017) 023006;

M. Kachelrieß, A. Neronov, D.V. Semikoz, Phys. Rev. D 97 (2018) 063011;

S. Profumo, J. Reynoso-Cordova, N. Kaaz, M. Silverman, Phys. Rev. D 97 (2018) 123008.

T. Linden, S. Profumo, Astrophys. J. 772 (2013) 18;

D. Hooper, P. Blasi, P.D. Serpico, JCAP (2009) 0901;JCAP 01 (2009) 025.

K. Ioka, Progr. Theoret. Phys. 123 (2010) 743.

[10] M.S. Turner, F. Wilczek, Phys. Rev. D 42 (1990) 1001;

J. Ellis, AIP Conf. Proc. 516 (2000) 21;

H. Cheng, J. Feng, K. Matchev, Phys. Rev. Lett. 89 (2002) 211301;

G. Kane, R. Lu, S. Watson, Phys. Lett. B 681 (2009) 151;

J. Feng, Ann. Rev. Astron. Astrophys. 48 (2010) 495;

C.H. Chen, C.W. Chiang, T. Nomura, Phys. Lett. B 747 (2015) 495;

H.C. Cheng, W.C. Huang, X. Huang, I. Low, Y.L. Sming Tsia, Q. Yuan , JCAP 03 (2017) 041;

Y. Bai, J. Berger, S. Lu, Phys. Rev. D 97 (2018) 115012.

J. Kopp, Phys. Rev. D 88 (2013) 076013. 